\title{
A hybrid of integer differential bees and flux balance analysis for improving succinate and lactate production
}

\begin{abstract}
The production of succinate and lactate from E.coli become a demand in pharmaceutical industries. To increase the yield of the production, gene knockout technique was implemented in various hybrid optimization algorithms. In recent years, several hybrid optimization have been introduced to optimize succinate and lactate production. However, the previous works were ineffective to produce the highest production due to the size and complexity of metabolic networks and the dynamic interaction between the components. Therefore, the main purpose of this study is to overcome the limitation of the existing algorithms which hybridizing Integer Differential Bees and Flux Balance Analysis (IDBFBA). The experimental results show a better performance in terms of growth rate and production yield of desired phenotypes compared to the method used in previous works.
\end{abstract}

Keyword: Flux balance analysis; Metabolic engineering; E.coli 